\title{
Does Perfusion MRI After Closed Reduction of Developmental Dysplasia of the Hip Reduce the Incidence of Avascular Necrosis?
}

\author{
Alex L. Gornitzky BS, Andrew G. Georgiadis MD, Mark A. Seeley MD, \\ B. David Horn MD, Wudbhav N. Sankar MD
}

Published online: 20 June 2015

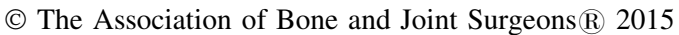

\begin{abstract}
Background Gadolinium-enhanced perfusion MRI (pMRI) after closed reduction/spica casting for developmental dysplasia of the hip (DDH) has been suggested as a potential means to identify and avoid avascular necrosis (AVN). To date, however, no study has evaluated the effectiveness of pMRI in clinical practice or compared it with other approaches (such as postreduction CT scan) to show a difference in the proportion of AVN.

Questions/purposes (1) Can a pMRI-based protocol be used immediately post closed reduction to minimize the risk that AVN would develop? (2) What are the overall hiprelated outcomes after closed reduction/spica casting using this protocol? (3) Do any patient-specific factors at the time of closed reduction predict future AVN?

Methods This was a retrospective cohort study at a large tertiary care children's hospital. Between 2009 and 2013
\end{abstract}

Each author certifies that he or she, or a member of his or her immediate family, has no funding or commercial associations (eg, consultancies, stock ownership, equity interest, patent/licensing arrangements, etc) that might pose a conflict of interest in connection with the submitted article.

All ICMJE Conflict of Interest Forms for authors and Clinical Orthopaedics and Related Research ${ }^{\mathbb{B}}$ editors and board members are on file with the publication and can be viewed on request.

Each author certifies that his or her institution approved the human protocol for this investigation and that all investigations were conducted in conformity with ethical principles of research. A waiver of consent/assent and a waiver of HIPAA authorization were approved by the author's institution before the start of this study.

A. L. Gornitzky, A. G. Georgiadis, M. A. Seeley, B. D. Horn, W. N. Sankar $(\square)$

Division of Orthopaedics, Children's Hospital of Philadelphia, 34th Street and Civic Center Boulevard, Philadelphia, PA 19104, USA

e-mail: sankarw@email.chop.edu we treated 43 patients with closed reduction/spica casting for $\mathrm{DDH}$, of whom $33(77 \%)$ received a postreduction pMRI. All patients were indicated for pMRI per treating surgeon preference. A convenience sample totaling 25 hips in 22 patients treated with pMRI was then established using the following exclusion criteria: DDH of neuromuscular/ syndromic origin, failed initial closed reduction, less than 1 year of clinical and radiographic followup, and subsequent open reduction. Next, the 40 patients treated with closed reduction between 2004 and 2009 were screened until the chronologically most recent 25 hips (after applying the previously mentioned exclusion criteria) were identified in 21 of the first 34 patients (62\%) screened. Although termed the CT group, specific postreduction imaging was not a defined inclusion criterion in this group with the majority (21 of 25 [84\%]) receiving postreduction CT and the remainder (four of 25 [16\%]) receiving only postreduction radiographs. All hips with globally decreased femoral head perfusion on postreduction pMRI were treated with immediate cast removal followed by repeat closed reduction or open reduction, as per surgeon preference, with two of $33(6 \%)$ requiring such further interventions. Salter criteria were then used to determine the proportion of AVN on radiographs at 1-year and final followup. Secondary outcomes including residual dysplasia and the need for further corrective surgery were ascertained through radiographic and retrospective chart review.

Results At 1-year followup there was no difference in the proportion of AVN in the historical CT group as compared with the pMRI group (six of 25 [24\%] versus one of 25 [4\%]; odds ratio [OR], 7.6; 95\% confidence interval [CI], $0.8-363 ; p=0.098)$. However, by final followup there was a statistically higher proportion of AVN in the CT group (seven of 25 [28\%] versus one of 25 [4\%]; OR, 9.3; 95\% CI, $1.0-438 ; \mathrm{p}=0.049)$. No patient with normal perfusion 
on postreduction pMRI went on to develop AVN. In those pMRI patients in whom a successful reduction was initially obtained, two of $25(8 \%)$ went on to require further corrective surgery and one of $25(4 \%)$ had a redislocation event. With the numbers available, no patient-specific factors at the time of closed reduction were predictive of future AVN, including the patient's age/weight, the presence of an ossific nucleus, history of previous bracing treatment, or the abduction angle in spica cast.

Conclusions A pMRI-based protocol immediately after closed reduction/spica casting may decrease the risk of AVN by helping the surgeon to evaluate femoral head vascularity. Although preliminary in nature, this study could serve to guide further investigation into the potential role of pMRI for the treatment of patients who require closed reduction/spica casting for DDH.

Level of Evidence Level III, therapeutic study.

\section{Introduction}

Developmental dysplasia of the hip (DDH) is one of the most common congenital deformities of the lower extremities. Patients failing initial conservative treatment with a Pavlik harness, abduction brace, or with a late diagnosis may be treated with closed reduction and spica casting of the hip. One of the primary complications associated with this treatment is avascular necrosis of the femoral head (AVN) with a reported incidence ranging widely from $3 \%$ to $60 \%[8,21]$.

Traditionally, plain radiographs or CT were used to verify a concentric reduction of the femoral head [31, 32]. However, these methods provide no information on femoral head vascularity. Furthermore, the radiographic signs used to diagnose AVN are only appreciable months to years after the presumed vascular insult. Reports have also investigated the potential influence of factors such as the ossific nucleus at the time of reduction or the hip abduction angle in the postoperative spica cast on AVN rate [27, 32], but these results are inconclusive.

Recent evidence has suggested MRI as a safe, effective, and reliable postreduction imaging modality [1, 5]. Gadolinium-enhanced perfusion MRI (pMRI) can provide the surgeon with data regarding vascular perfusion to the femoral head $[2,13]$. To date, however, no study has evaluated the effectiveness of pMRI as a primary decision-making tool or compared it with other approaches (such as postreduction CT scan) to show a difference in AVN rate or other clinical outcomes. As a result of the long-term disability associated with AVN, and the mixed success of closed reduction [6, 18, 24], the role of closed reduction/spica casting in the management of DDH has recently been questioned. If a pMRI-based protocol proves helpful, its use might allow the surgeon to critically evaluate the quality of reduction while simultaneously minimizing the risk for future AVN and therefore improve the results of closed reduction procedures.

We therefore asked: (1) Can a pMRI-based protocol be used immediately post closed reduction to minimize the risk that AVN would develop? (2) What are the overall hiprelated outcomes after closed reduction/spica casting using this protocol? (3) Do any patient-specific factors at the time of closed reduction predict future AVN?

\section{Patients and Methods}

This was a nonmatched retrospective comparative study at a large tertiary care children's hospital. Our institutional review board approved this study. Potential cases were ascertained using the Current Procedural Terminology (CPT) code for closed reduction for DDH (27257) between 2001 and 2013. A pMRI protocol was first instituted at our hospital in 2009. Between 2009 and 2013, 43 patients were treated with closed reduction/spica casting for $\mathrm{DDH}$, of whom $33(77 \%)$ received a postreduction pMRI. All patients were indicated for pMRI per treating surgeon preference with reasons for no pMRI including late adoption of the protocol (two), vascular access problems (two), renal disease (one), age older than 2 years (three), and inconsistent protocol use (two). A convenience sample totaling 25 hips in 22 of 33 patients $(66 \%)$ treated with pMRI was then established using the following exclusion criteria: DDH of neuromuscular/syndromic origin, failed previous closed reduction, less than 1 year of clinical and radiographic followup, and subsequent open reduction. Next, the 40 patients treated with closed reduction between 2004 and 2009 were screened until the chronologically most recent 25 hips meeting the same exclusion criteria were identified. In total, 25 hips in 21 patients (of the first 34 screened [62\%]) were identified for the CT cohort (Fig. 1). Although termed the CT group, specific postreduction imaging was not a defined inclusion criteria in this group with the majority (21 of 25 [84\%]) receiving postreduction CT and the remainder (four of 25 [16\%]) receiving only postreduction radiographs. On average, patients in the CT group received their followup imaging approximately 14 days (range, $0-28$ days) after reduction.

Followup in both groups was defined as an in-person clinical examination and review of radiographs. Excluded patients with less than 1 year of followup included three of 43 patients (7\%) in the pMRI group and four of $34(12 \%)$ in the CT group. As a result of the potential confounding effect of different treatment methods on the development of AVN, patients who were advanced to open reduction, whether for unsuccessful closed reduction or global decreased enhancement on pMRI, were noted but not 


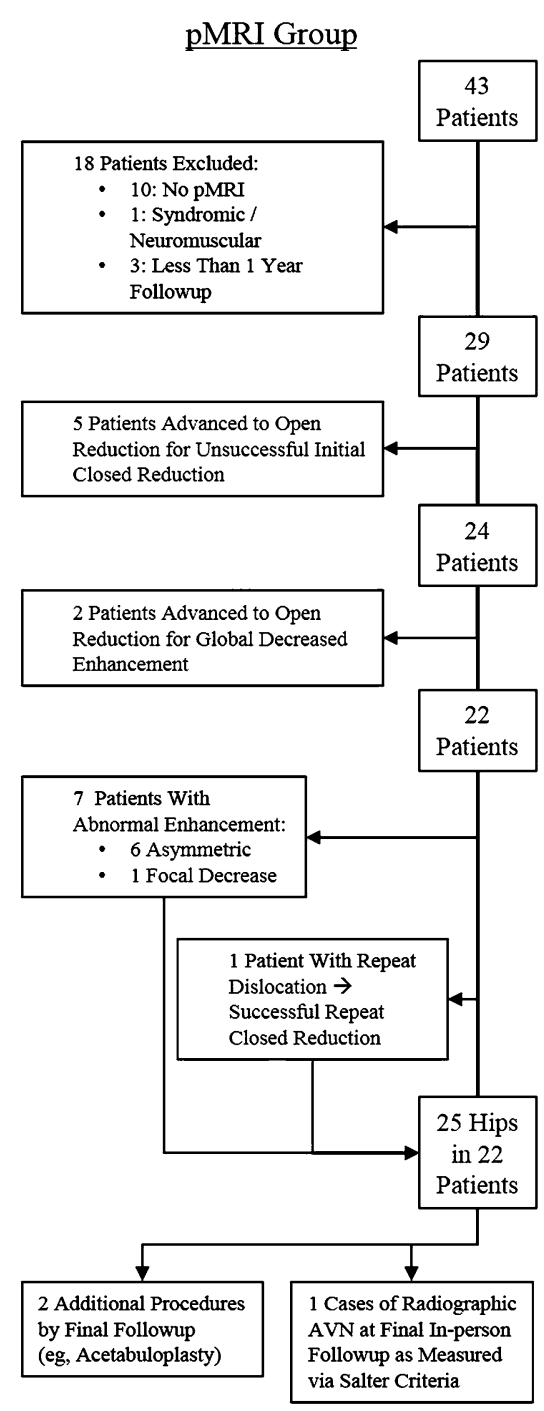

A

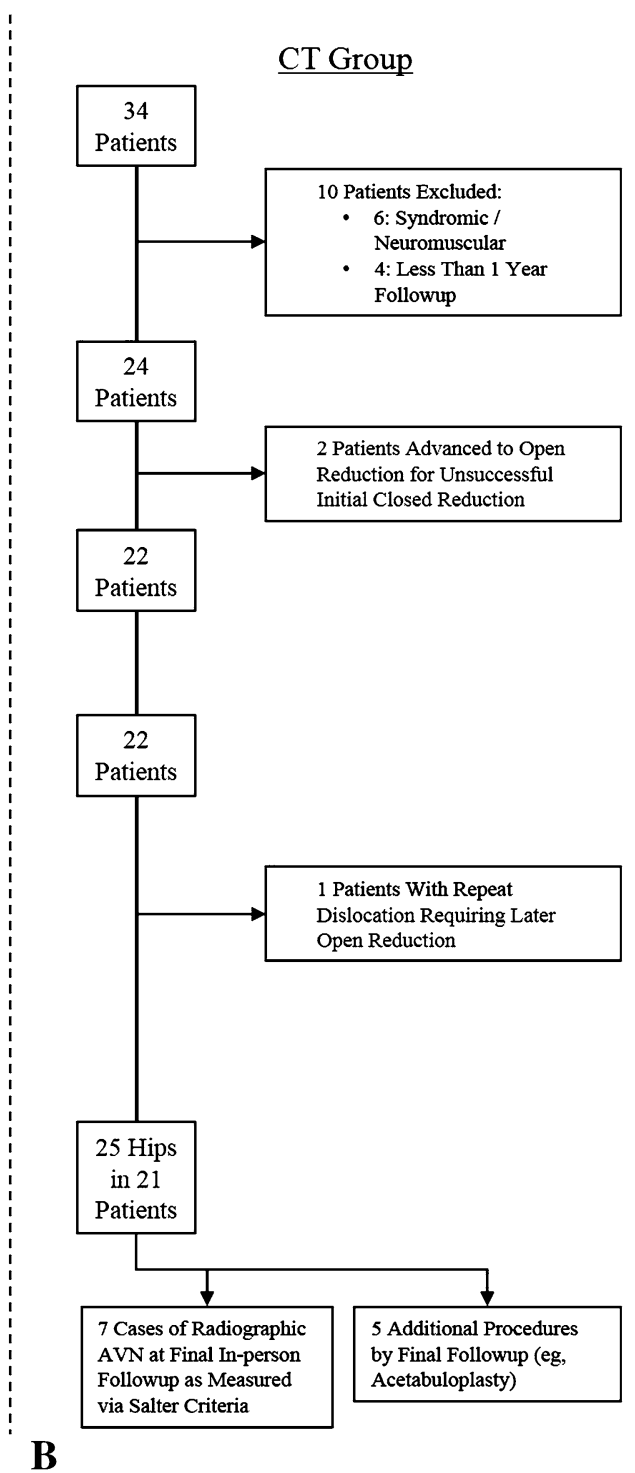

Fig. 1A-B This flowchart depicts evaluation, exclusion, enrollment, and outcomes in the pMRI group (A) and the CT group (B).

further evaluated for the development of radiographic AVN (Fig. 1). Gender, laterality, previous treatment with a Pavlik harness and/or abduction brace, concurrent adductor tenotomy, abduction angle in a spica cast, and length of followup were not significantly different between the two groups. Children in the pMRI group were older, heavier, and more likely to have an ossific nucleus present at the time of reduction than children in the CT group (Table 1).

Standard treatment at our institution for patients with DDH who have failed Pavlik harness and/or abduction brace treatment, or are diagnosed late, includes an attempt at closed reduction and spica casting. Traction is not used and adductor tenotomy is performed at the discretion of the operating surgeon. Routine cast changes under general anesthesia allow for incremental growth with subsequent postreduction imaging obtained, per surgeon preference, to confirm maintained reduction. Total casting time averages 3 months, after which children are placed into nighttime abduction orthoses until 2 years of age.

Perfusion MRI was performed soon postoperatively without sedation (for infants younger than 9 months of age) or immediately after cast placement under the same anesthetic (for those older than 9 months of age) according to the protocol of Jaramillo et al. [14]. All studies were performed within 6 hours of closed reduction to allow for timely cast removal before the presumed onset of irreversible ischemic changes [12]. Coronal T2 and axial fatsuppressed $\mathrm{T} 1$ images were obtained before gadolinium, and repeated axial fat-suppressed fast $\mathrm{T} 1$ sequences and postcontrast subtraction images were obtained afterward.

The on-call board-certified pediatric musculoskeletal radiologists reviewed the pMRI at the time of the scan and 
Table 1. Baseline characteristics stratified by cohort

\begin{tabular}{|c|c|c|c|c|c|}
\hline Variable & $\mathrm{CT}(\mathrm{n}=25)$ & $\mathrm{pMRI}(\mathrm{n}=25)$ & Odds ratio* & Difference $^{\dagger}$ & p value \\
\hline Gender & & & $6(0.6-295)$ & & 0.189 \\
\hline Male $(\%)$ & $5(20)$ & $1(4)$ & & & \\
\hline Female $(\%)$ & $20(80)$ & $24(96)$ & & & \\
\hline Affected side & & & $0.8(0.2-4)$ & & 1.000 \\
\hline Left $(\%)$ & $18(72)$ & $19(76)$ & & & \\
\hline Right $(\%)$ & $7(28)$ & $6(24)$ & & & \\
\hline Bilateral hip involvement & $3(12)$ & $4(16)$ & $0.7(0.1-5)$ & & 0.698 \\
\hline Age at reduction, median months (range) & $5(3-16)$ & $8(4-19)$ & & -3 & $<0.001$ \\
\hline Weight at reduction, median kilograms (range) & $6(5-12)$ & $8(6-10)$ & & -1 & 0.009 \\
\hline Presence of ossific nucleus at reduction (\%) & $9(36)$ & $17(68)$ & $0.3(0.1-0.98)$ & & 0.047 \\
\hline Previous treatment: either Pavlik or abduction brace (\%) & $19(76)$ & $12(48)$ & $3.4(0.9-14)$ & & 0.079 \\
\hline Previous treatment: abduction brace after failed Pavlik (\%) & $5(26)$ & $8(67)$ & $0.2(0.03-1.1)$ & & 0.060 \\
\hline Adductor tenotomy (\%) & $8(32)$ & $13(52)$ & $0.4(0.1-2)$ & & 0.252 \\
\hline Abduction angle, mean degrees (SD) & $54( \pm 7)$ & $54( \pm 7)$ & & $-1(-5$ to 4$)$ & 0.749 \\
\hline 1-year followup, median months (range) & $13(10-28)$ & $12(11-20)$ & & 0 & 0.786 \\
\hline Final followup, median months (range) & $36(17-47)$ & $30(13-49)$ & & 7 & 0.118 \\
\hline
\end{tabular}

* Odds ratio (95\% confidence interval) for CT with respect to pMRI; ${ }^{\dagger}$ mean difference (95\% confidence interval) or difference of medians for CT group compared with pMRI group; ${ }^{*}$ p values correspond to intergroup differences based on the Fisher's exact test for categorical variables, Student's t-test for normally distributed continuous variables, and Mann-Whitney U-test for nonnormally distributed continuous variables; pMRI = perfusion MRI.

Table 2. Criteria for evaluation of pMRI findings according to Tiderius et al. [34]

\begin{tabular}{ll}
\hline Enhancement & Description \\
\hline Normal & $\begin{array}{l}\text { Full visualization of the epiphyseal vascular canals } \\
\text { and physis }\end{array}$ \\
Asymmetric & $\begin{array}{l}\text { Either the epiphyseal vascular canals or the physes had } \\
\text { reduced intensity compared with the contralateral } \\
\text { side or the overall signal intensity of the epiphyseal } \\
\text { cartilage was reduced compared with the } \\
\text { contralateral side }\end{array}$ \\
$\begin{array}{c}\text { Focal } \\
\text { decrease }\end{array}$ & $\begin{array}{l}\text { Clear area of decreased signal intensity within the } \\
\text { epiphys or the ossification center }\end{array}$ \\
Global & $\begin{array}{c}\text { Absence of enhancement within the entire epiphysis, } \\
\text { including the ossification center if present }\end{array}$ \\
\hline
\end{tabular}

pMRI = perfusion MRI.

conveyed the results to the treating surgeon (BDH, WNS, DAS, JMF) to allow for real-time decision-making regarding the quality of reduction and femoral head perfusion. Vascular perfusion to the femoral head was categorized according to the four-level scale described by Tiderius et al. (Table 2) [34] (examples of each pMRI finding are depicted in Fig. 2). Asymmetric or focally decreased perfusion was managed with no change in treatment; globally decreased perfusion or persistently dislocated hips were managed with immediate spica cast removal. Patients were then scheduled for repeat closed reduction or progression to open reduction at a later date, per surgeon preference. In all, two patients had globally decreased enhancement and were subsequently removed from their cast and excluded from further outcome analysis: one had open reduction after a second attempt at closed reduction and the second was advanced directly to open reduction (Table 3 ).

Abduction angles were measured on the mid axial slice of the first postreduction advanced imaging modality (CT or MRI) as the angle between the main femoral axis and the midsagittal line. The presence or absence of the ossific nucleus at the time of reduction was assessed by preoperative ultrasound and, in the absence of this or an immediate preoperative radiograph, by postoperative CT/MRI.

The primary outcome was assessed according to the radiographic signs of AVN as described by Salter et al. [25] (Table 4) (some examples are depicted in Fig. 3). Similar to previous studies, transient Grade 1 changes were regarded as clinically insignificant and not classified as AVN [19]. AP pelvis plain radiographs were independently reviewed by an orthopaedic fellow (MAS) blinded to MRI results and clinical history. Findings were subsequently verified in a blinded fashion by the senior author (WNS). As a result of the more recent implementation of the pMRI-based protocol, and to facilitate uniformity of followup length between the groups, final followup was capped at less than or equal to 4 years post-reduction.

Measures to evaluate secondary outcomes included the acetabular index (AI) for residual dysplasia and the 

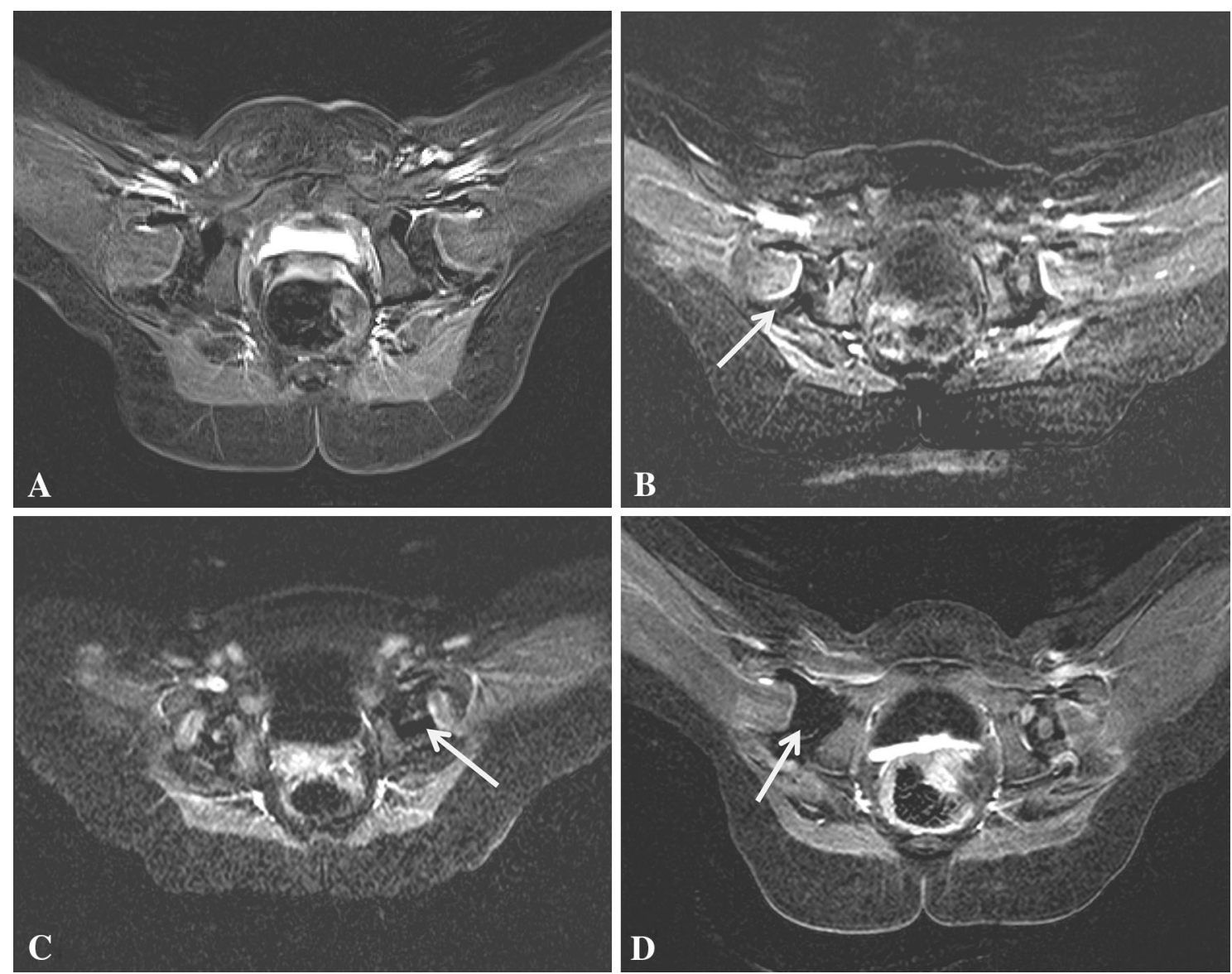

Fig. 2A-D Representative perfusion-MRI findings in four patients. Images are $\mathrm{T} 1$ axial post-gadolinium subtraction sequences, all following closed hip reduction and spica casting. (A) Symmetric epiphyseal vascular channels after left hip reduction. (B) Asymmetric

International Hip Dysplasia Institute criteria (Fig. 4) [22] for hip location. These outcomes were measured in a blinded fashion simultaneously to the evaluation for AVN. Remaining secondary outcomes, including the need for additional surgical correction or redislocation events, were assessed through retrospective chart review.

\section{Statistical Analysis}

Two-tailed Fisher's exact test was used to determine the strength of the relationships between categorical variables. Continuous variables were analyzed by the Shapiro-Wilk test to assess for normality. Normal variables were analyzed using independent t-tests; nonnormal variables were assessed using the Mann-Whitney $U$ test. Categorical variables are reported as proportions (numerator/denominator and percentage) with their associated effect size (odds ratio $[\mathrm{OR}]$ ) and $95 \%$ confidence interval (CI). All continuous variables are presented with a measure of central tendency (mean or median), spread (SD or range),

vascular enhancement of the right epiphysis compared to the left side (arrow). (C) Focal, central, epiphyseal non-enhancement of left hip (arrow). (D) Global decreased perfusion of the right epiphysis (arrow).

and difference (mean difference [MD] with 95\% CI or difference of medians) as determined based on fit with a normal distribution. An alpha level of 0.05 was used in all tests. Statistical analysis was performed using Stata (StataCorp, College Station, TX, USA).

\section{Results}

At 1-year followup there was no difference in the proportion of AVN in the historical CT group as compared with the pMRI group (six of 25 [24\%] versus one of 25 [4\%]; OR, 8; 95\% CI, 0.8-363; p = 0.098). However, by final followup there was a statistically higher proportion of AVN in the CT group (seven of 25 [28\%] versus one of 25 [4\%]; OR, 9; 95\% CI, 1.0-438; $\mathrm{p}=0.049$ ) (Table 5). The absolute risk reduction in the pMRI group at final followup was $24 \%(95 \%$ CI, 5\%-43\%) and the number needed to treat with pMRI to prevent one case of AVN was five $(95 \% \mathrm{CI}$, 2-21). No patient developed AVN after 24 months of followup and, as per our inclusion criteria, all patients 


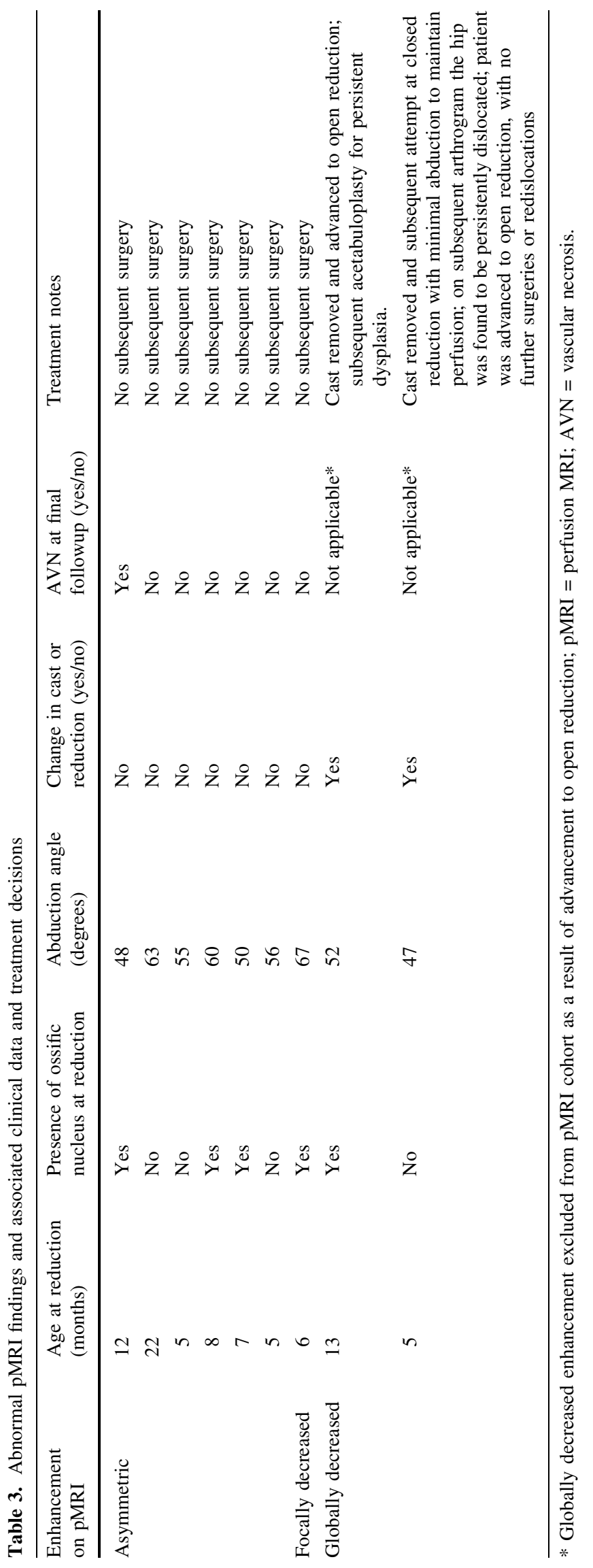


classified as having AVN had Salter 2, 3, 4, or 5 changes. Abnormal enhancement on pMRI was not associated with an increased abduction angle $\left(55^{\circ} \pm 7^{\circ}\right.$ [normal pMRI] versus $53^{\circ} \pm 7^{\circ}$ [abnormal enhancement]; MD $2^{\circ} ; 95 \% \mathrm{CI}$, $-4^{\circ}$ to $\left.8^{\circ} ; p=0.438\right)$. In patients who were maintained in a spica cast after asymmetric or focally decreased pMRI enhancement $(\mathrm{n}=7$ [28\%]), one (with asymmetric

Table 4. Salter's criteria for AVN [25]

\section{Grade Description}

1 Absence of the ossific nucleus within 1 year of procedure

2 Absence of growth in the ossific nucleus within 1 year of procedure

3 Broadening of the femoral neck/metaphysis within 1 year of procedure

$4 \quad$ Fragmentation or sclerosis of the femoral head

5 Residual femoral head or neck deformity, including coax magna, vara, valga, or plana

$\mathrm{AVN}=$ avascular necrosis enhancement) went on to develop AVN. Conversely, no patient with a normal pMRI after closed reduction developed AVN.

There was no significant difference in residual dysplasia in the CT versus the pMRI group with the numbers available as measured by the $\mathrm{AI}$ at 1 -year $\left(29^{\circ} \pm 4^{\circ}\right.$ versus $28^{\circ} \pm 5^{\circ} ; \mathrm{MD} 1^{\circ} ; 95 \% \mathrm{CI},-1^{\circ}$ to $3^{\circ} ; \mathrm{p}=0.383$ ) and final followup $\left(25^{\circ} \pm 4^{\circ}\right.$ versus $25^{\circ} \pm 5^{\circ}$; MD $-1^{\circ} ; 95 \% \mathrm{CI}$, $-3^{\circ}$ to $2^{\circ} ; \mathrm{p}=0.543$ ) or the number of patients having an abnormal International Hip Dysplasia Institute grade above one at 1-year (eight of 25 [32\%] versus three of 25 [12\%]; OR, 4; 95\% CI, 0.7-23; $\mathrm{p}=0.171$ ) or final followup (three of 25 [12\%] versus one of 25 [4\%]; OR, 3; 95\% CI, $0.2-179 ; p=0.609)$ (Table 5). One patient out of $21(5 \%)$ in the CT group redislocated and required later open reduction. One patient out of $22(5 \%)$ in the pMRI group was found to be redislocated at subsequent cast change and underwent successful repeat closed reduction. Five hips out of 25 (20\%) in the CT group underwent further corrective surgery (eg, pelvic and/or femoral osteotomies) versus two
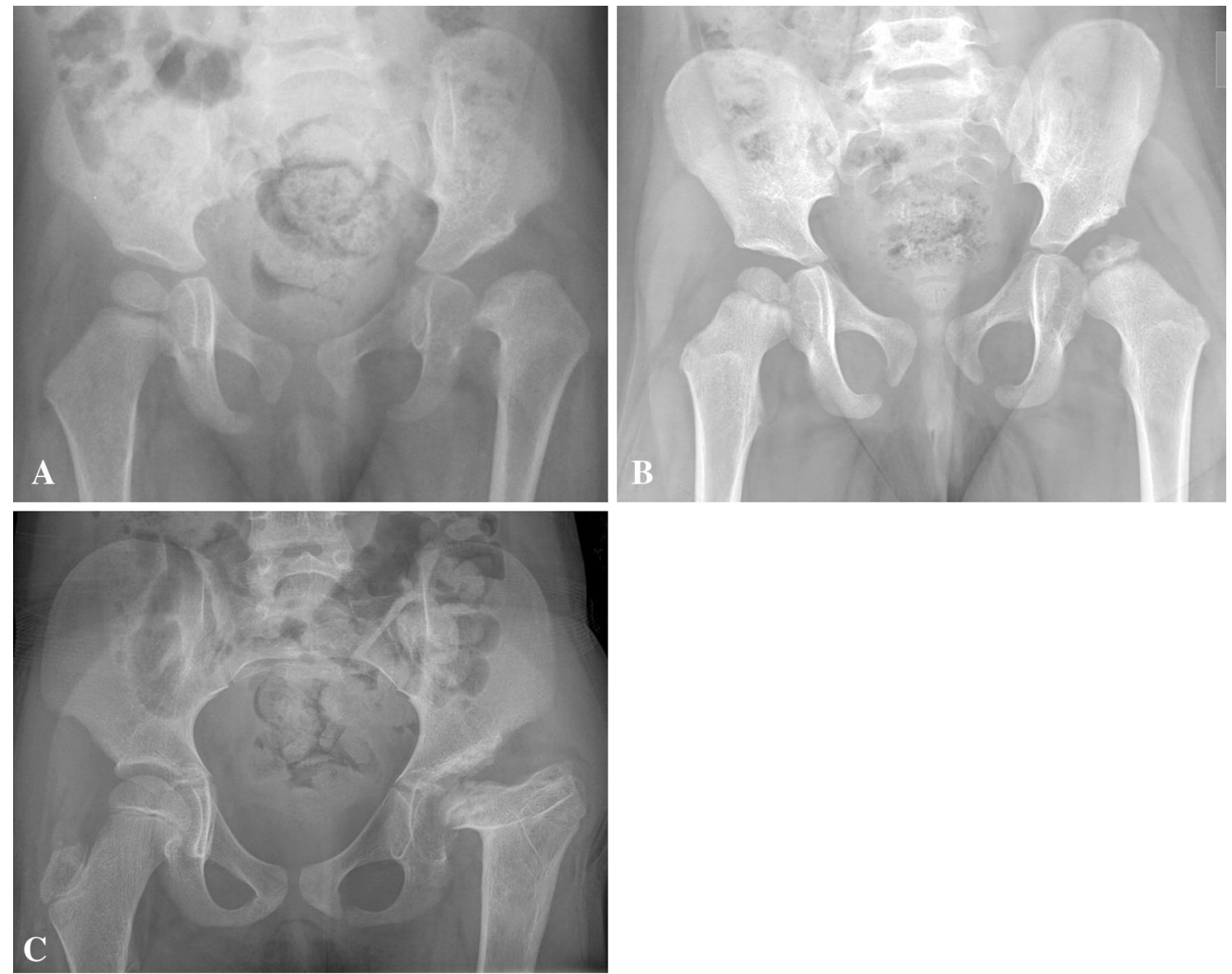

Fig. 3A-C Representative images of some hips meeting Salter criteria for AVN. (A) Salter 3-broadening of the femoral neck/metaphysis within 1 year of procedure (B) Salter 4-fragmentation of the femoral head. (C) Salter 5-residual head/neck deformity. 
hips out of $25(8 \%)$ in the pMRI group (OR, 3; 95\% CI, $0.4-33 ; \mathrm{p}=0.417$ ).

With the numbers available, patients who developed AVN at final followup $(n=8[16 \%])$ did not differ in terms of gender, laterality, previous treatment with a Pavlik

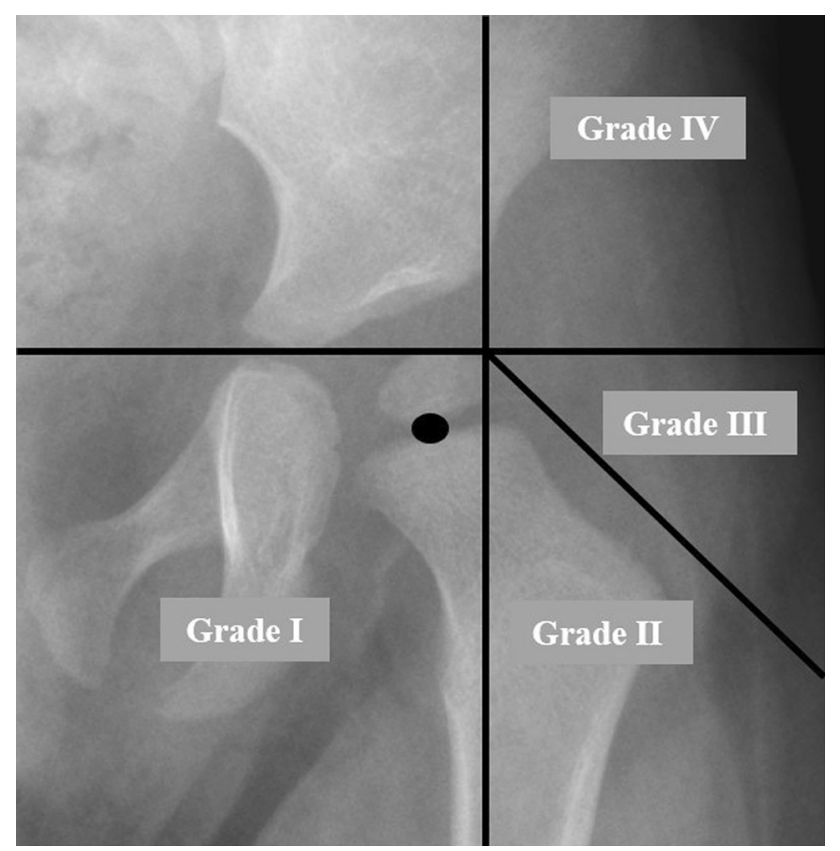

Fig. 4 The International Hip Dysplasia Institute (IHDI) criteria are a means of classifying DDH disease severity even when the ossification center is absent [22]. To start, Hilgenreiner's line (H-line) is drawn through the top of the triradiate cartilages bilaterally. Next, Perkin's line (P-line) is drawn at the superolateral margin of the acetabulum perpendicular to the $\mathrm{H}$-line. A diagonal line is then drawn $45^{\circ}$ inferolaterally from the junction of the $\mathrm{H}$-line and P-line. Finally, based on the location of the midpoint of the superior margin of the ossified metaphysis, as shown here with a black oval, the residual deformity may be graded from 1 to 4 . In this example, the patient has an IHDI grade of 1 . harness and/or abduction brace, concurrent adductor tenotomy, or age/weight at reduction from those who did not develop AVN ( $n=42$ [84\%]; Table 6). We did not find a statistically significant difference in abduction angle between those patients who developed AVN and those who $\operatorname{did} \operatorname{not}\left(50^{\circ} \pm 6^{\circ}[\mathrm{AVN}]\right.$ versus $55^{\circ} \pm 7^{\circ}$ [no AVN]; MD $-5^{\circ} ; 95 \% \mathrm{CI},-10^{\circ}$ to $\left.0^{\circ} ; \mathrm{p}=0.065\right)$. Although fewer patients in the CT group had an ossific nucleus present at the time of reduction ( $36 \%$ versus $68 \%$; OR, $0.3 ; 95 \% \mathrm{CI}$, $0.07-0.98 ; \mathrm{p}=0.047$ ) (Table 1 ), the presence of the ossific nucleus was not, with the numbers available, by itself predictive of future AVN (25\% versus 57\%; OR, 0.3; $95 \%$ CI, 0.02-2; $\mathrm{p}=0.132$ ) (Table 6).

\section{Discussion}

With an incidence of approximately 1.5 to 20 per 1000 births, of which one to two per 10,000 present late, DDH is one of the most common congenital deformities of the lower limb [10,29]. Avascular necrosis of the femoral head is a devastating complication after closed reduction $[3,15]$ potentially resulting in early degenerative joint disease and impaired function $[3,9,15,20]$. To date, no factors have been shown as reliable predictors of future AVN. Although previous studies $[14,34]$ have described the use of pMRI in patients with $\mathrm{DDH}$, to our knowledge, this is the first to report on its performance as a primary clinical decisionmaking tool or compare it with other approaches such as postreduction CT. Using a nonmatched retrospective comparative cohort, we demonstrated a reduction in risk of developing AVN when patients were treated under a pMRI-based protocol compared with a historical cohort evaluated with CT. Furthermore, there were no differences in overall hip-related outcomes, including redislocation events or the need for further corrective surgery, and based

Table 5. Outcome measurements stratified by cohort

\begin{tabular}{|c|c|c|c|}
\hline Variable & pMRI $(n=25)$ & $\mathrm{CT}(\mathrm{n}=25)$ & $\mathrm{p}$ value* \\
\hline \multicolumn{4}{|l|}{ 1-year followup } \\
\hline IHDI grade, number of hips greater than $1(\%)$ & $3(12)$ & $8(32)$ & 0.171 \\
\hline Acetabular index, mean degrees (SD) & $28.0( \pm 4.6)$ & $29.1( \pm 3.7)$ & 0.384 \\
\hline $\operatorname{AVN}(\%)$ & $1(4)$ & $6(24)$ & 0.098 \\
\hline \multicolumn{4}{|l|}{ Final followup } \\
\hline IHDI grade, number of hips greater than $1(\%)$ & $1(4)$ & $3(12)$ & 0.609 \\
\hline Acetabular index, mean degrees (SD) & $25.4( \pm 4.6)$ & $24.6( \pm 4.2)$ & 0.543 \\
\hline $\operatorname{AVN}(\%)$ & $1(4)$ & $7(28)$ & 0.048 \\
\hline Further corrective surgery, number of hips (\%) & $2(8)$ & $5(20)$ & 0.417 \\
\hline Redislocation following successful initial closed reduction, number of hips $(\%)^{\dagger}$ & $1(4)$ & 0 & 1.000 \\
\hline
\end{tabular}

* $\mathrm{p}$ values correspond to intergroup differences based on the Fisher's exact test for categorical variables and Student's t-test for continuous variables; 'patient retained in pMRI group for further analysis after successful repeat closed reduction; pMRI = perfusion MRI; IHDI = International Hip Dysplasia Institute criteria; AVN = avascular necrosis. 
Table 6. Baseline characteristics stratified by final AVN status

\begin{tabular}{lcc}
\hline Variable & AVN $(\mathrm{n}=8)$ & No AVN (n=42) \\
\hline Gender & & $\mathrm{p}$ value* \\
$\quad$ Male $(\%)$ & $1(13)$ & $5(12)$ \\
Female (\%) & $7(87)$ & $37(88)$ \\
Affected side & $6(75)$ & $31(74)$ \\
Left (\%) & $2(25)$ & $11(26)$ \\
Right (\%) & $5(4-7)$ & $7(5-10)$ \\
Age at reduction, median months (IQR) & $6.1(5.9-7.3)$ & $7.3(6.4-8.7)$ \\
Weight at reduction, median kilograms (IQR) & $2(25)$ & $24(57)$ \\
Presence of ossific nucleus at reduction (\%) & $5(63)$ & $26(62)$ \\
Previous treatment: either Pavlik or abduction brace (\%) & $1(20)$ & $12(46)$ \\
Previous treatment: abduction brace after failed Pavlik (\%) & $4(50)$ & $17(40)$ \\
Adductor tenotomy (\%) & $49.8( \pm 5.8)$ & 0.218 \\
Abduction angle, mean degrees (SD) & $54.8( \pm 7.0)$ \\
\hline
\end{tabular}

* p values correspond to intergroup differences based on the Fisher's exact test for categorical variables, Student's t-test for normally distributed continuous variables, and Mann-Whitney U test for nonnormally distributed continuous variables; AVN = avascular necrosis; IQR = interquartile range.

on our data, there were no patient-specific factors at the time of closed reduction that were predictive of future AVN.

Our study has a number of limitations. The data were collected retrospectively from a single, urban institution. It should be noted that patients treated under the pMRI-based protocol were older, heavier, and more likely to have had an ossific nucleus visible at the time of reduction, all of which likely represent similar markers of physiologic maturity. Before ossification, the vasculature within the femoral epiphysis may be more vulnerable to compression and ischemia [6, 28]. Although our small numbers failed to detect a relationship between the presence of an ossific nucleus and the future development of AVN, the younger age at treatment may have contributed to the CT group's increased proportion of AVN. It is unclear why there was a difference in age between the cohorts. It is possible that referral patterns for patients with late-diagnosed DDH changed or that the senior author's evolution toward the use of abduction bracing as an option before closed reduction led to older ages at the time of this procedure. However, in the latter scenario, those failing bracing in the more recent pMRI group would likely represent worse disease and would therefore be expected to have worse outcomes and a higher proportion of AVN rather than a lower one. Regardless, the senior authors are advocates of closed reduction and have refined their protocols to preserve its role in the treatment of DDH. Therefore, it is less likely that an increase in open reduction as the first option for severe disease contributed to our observation of a lower proportion of AVN in the pMRI group. Addressing biases, an approximately equal proportion of patients in both groups were excluded for loss to followup, making it less likely that transfer bias impacted our findings. Additionally, because this study used convenience sampling, selection bias may have been introduced if patients in either group were not included, which could have affected the proportion of detected AVN in either direction.

Other limitations of this study include the inherent properties of perfusion MRI. Studies have shown that not all patients with a global decrease in perfusion go on to develop AVN, whereas some patients who show only minimal asymmetries in enhancement do [34]. Nevertheless, in this study we aimed to evaluate the implementation of a pMRI-based protocol derived from Tiderius et al.'s [34] findings regarding globally decreased enhancement, not the use of pMRI itself. Consequently, although the subjectivity of pMRI interpretation may have affected both treatment and outcomes, each pMRI did not undergo repeat evaluation for classification or determination of inter- or intraobserver reliability, because it was felt that any variability in the reading applied retrospectively would not reflect an accurate assessment of the applied protocol at the time of actual treatment. AVN itself is also a difficult concept to define. No universal diagnostic criteria exist, and it may take several years for patients to demonstrate radiographic evidence of AVN [3]. Although Salter criteria are a well-accepted evaluation tool to detect radiographic AVN [19, 34] and we used blinded evaluation to classify radiographs, subjective radiographic interpretation may have impacted our findings. Although a lower proportion of patients treated under the pMRI-based protocol developed AVN, the small study size precluded the use of regression analysis to control for potentially confounding variables. 
This included an in-depth evaluation of predictors of future AVN, because only eight patients developed AVN, or a subgroup analysis of those with bilateral dysplasia, which may indicate more severe disease. Additionally, we only evaluated followup to a maximum of 4 years. Although no patient developed AVN after 2 years, a longer followup may result in the discovery of additional patients developing AVN and undergoing additional corrective surgery $[15,20]$ or improved detection of mild residual dysplasia. Ideally, patients would be followed to skeletal maturity. In the current study, however, we chose to report AVN rates at two shorter, well-defined intervals to provide consistent comparisons between cohorts and a reasonable length of followup for future comparative studies. Finally, as a result of the limited number of patients that require closed reduction for DDH, we were only able to report on a small number of patients resulting in borderline statistical significance with wide CIs. Although we believe in the importance of stimulating discussion and further research on such a potentially helpful tool, it must also be considered that only one or two changed outcomes in either group could have negated the findings. Therefore, these results must be considered preliminary and further validated through prospective investigation.

As reported by Tiderius et al. [34], pMRI shows promise as a means of potentially identifying early ischemia responsible for AVN at a time when the avascular changes in the femoral head are still reversible and alternative treatment options exist. In their cohort, a global decreased enhancement on pMRI correlated strongly with the future development of AVN independent of age or abduction angle. In their review of the literature, Cooper et al. [8] found a wide range in reported AVN rates between $3 \%$ and $60 \%$, which they attributed to variations in the definition of AVN and the length of followup. In a more recent sample $[4,6,7,16,19,21,24,30,34]$, AVN rates varied more consistently between $7 \%$ and $36 \%$, but again a wide variety of radiographic criteria was used to identify AVN. In this study, we used Salter criteria as did Tiderius et al. [34]. Overall, our $4 \%$ incidence of AVN in the pMRI group is comparable to the lowest of any previously reported results. Although there also appeared to be a trend toward lower AVN in the pMRI group at 1 year, potentially as a result of the small sample size, we were unable to detect a statistically significant difference at this time point. Consistent with previous findings [34], no patient with a normal postreduction pMRI in our series went on to develop AVN. Although one patient with asymmetric enhancement developed AVN, our protocol mandated that only those with a global decrease in vascular enhancement undergo cast removal and change in treatment course. Accordingly, the significance of asymmetric enhancement still needs to be elucidated. Furthermore, although the senior authors have been successful with repeat closed reduction following globally decreased perfusion after the first attempt (Fig. 5), a well-designed, prospective study will be required to validate these findings. Finally, confirming earlier reports on the modality's safety [26], no child had an adverse complication linked to pMRI.

Failure of closed reduction is linked with a sixfold increase in the annual risk of additional corrective surgery [11]. In their review of closed reductions, Cooper et al. [8] found reported rates of failed reduction between $0 \%$ and $25 \%$ and redislocation rates between $3 \%$ and $14 \%$. In the present study, five of 43 patients $(12 \%)$ screened for inclusion into the pMRI group were unable to be concentrically reduced on the initial attempt. Of those who were reduced successfully, one went on to redislocate and required repeat closed reduction. A concentric reduction of the hip is also crucial for continued acetabular growth and development [16]. Consistent with prior studies, both of our cohorts showed continued improvement of their dysplasia as measured by AI and International Hip Dysplasia Institute criteria for approximately 3 to 4 years after closed reduction $[3,16,33,36]$. We saw no differences between the groups in terms of initial or final acetabular dysplasia. Finally, residual dysplasia may increase the use of additional surgical interventions. Previously reported rates of further corrective surgery have varied between $13 \%$ and $76 \%[6,7,18,21,37]$ as a result of differences in individual surgeons' practices. Although our results compare favorably with those studies, our reported overall rate of $16 \%$ (12\% in the pMRI group) likely underrepresents the true value as a result of a shorter duration of followup.

Numerous reports have sought to identify predictive factors at the time of reduction associated with the future development of AVN. Although increasing hip abduction in the spica cast improves stability, it may also lead to increased vascular compromise [14, 19, 25] with positional extremes causing increased extracapsular compression of the blood supply to the developing femoral head [23]. Clinical studies, however, have been mixed [19, 32, 34]. We found no difference in abduction angle with the numbers available in either those who developed AVN or those displaying abnormal enhancement on pMRI. Consistent with prior studies, gender [3], laterality [3], previous treatment with a Pavlik harness/abduction brace [3, 18, 24], and concurrent procedure [18] all had no effect on final AVN rates with the numbers available in our cohort. The presence or absence of the ossific nucleus at the time of reduction remains a controversial issue. In our study, patients in the CT group were older, heavier, and more likely to have had an ossific nucleus present at the time of reduction. However, with our small numbers, the ossific nucleus was not by itself a predictor of AVN. Clinical studies have remained mixed on the importance of the 

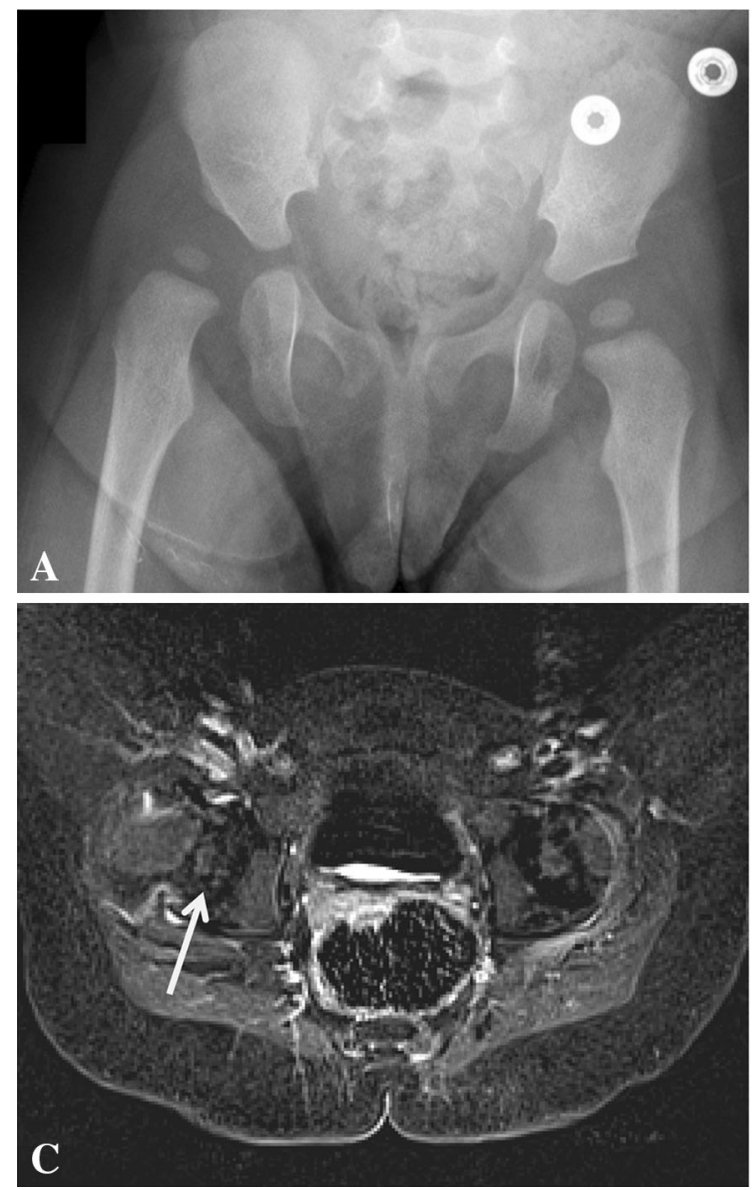

Fig. 5A-D The following is a case example of a pMRI-altered treatment course. (A) A 9-month-old girl had right hip DDH as demonstrated on AP pelvis radiograph. She subsequently underwent arthrogram, closed reduction, and spica casting. (B) The immediate postoperative pMRI demonstrated globally decreased perfusion of her right femoral epiphysis (arrow). Her cast was immediately removed and she had a repeat arthrogram and closed reduction 1 week later, at which time she was casted into less abduction. (C) Postreduction

ossific nucleus. Although many studies looking at both open and closed reduction have shown a protective response with respect to its presence [4, 6, 27], studies looking more specifically at closed reduction have shown no effect [7, 18, 30, 35]. Some studies have linked older age at treatment to an increased risk of AVN [30, 35] and an increased rate of residual dysplasia [16], whereas others have concluded younger age has either no influence $[3,16$, $18,27]$ or a negative effect on AVN rates [15, 25]. Furthermore, early reduction takes advantage of the acetabulum's increased remodeling potential at young ages $[18,23,27]$ and may improve clinical outcomes [20] and reduce the need for future corrective surgeries [17]. Even if our improved AVN rates were the result of both the pMRI itself and inadvertently delayed intervention, we are still
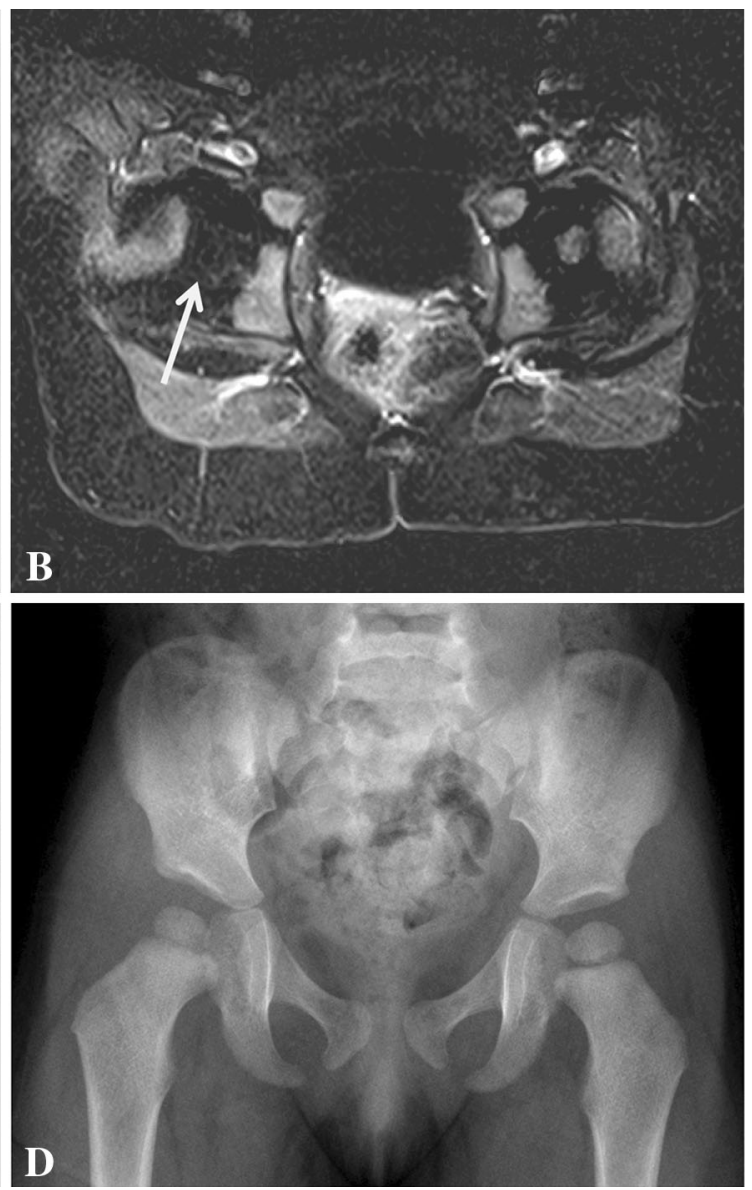

pMRI following the second attempt demonstrated symmetric enhancement of the vascular canals throughout the entire epiphysis and of the secondary ossification center (arrow). (D) Most recent AP pelvis radiograph demonstrates excellent interval femoral head development without signs of AVN. Of note, this patient is not included in the reported pMRI cohort as she was treated after the study's timeframe.

reporting results favorable to the lowest AVN rates that have been previously reported.

A pMRI-based protocol immediately after closed reduction/spica casting may decrease the risk of AVN by helping the surgeon to evaluate femoral head vascularity and adjust treatment before the onset of irreversible avascular changes to the epiphysis. Although our data is encouraging, as a result of the limitations described, these results should be considered preliminary and further validated in prospective, comparative trials before widespread clinical application.

Acknowledgments We thank Carolyn J. Winslow for her assistance with statistical analysis. We also thank David A. Spiegel MD, and John M. Flynn MD, for contributing patients to both the CT and pMRI cohorts. 


\section{References}

1. Bachy M, Thevenin-Lemoine C, Rogier A, Mary P, Pointe DL, Vialle R. Utility of magnetic resonance imaging (MRI) after closed reduction of developmental dysplasia of the hip. J Child Orthop. 2012;6:13-20.

2. Barnewolt CE, Shapiro F, Jaramillo D. Normal gadolinium-enhanced MR images of the developing appendicular skeleton: Part I. cartilaginous epiphysis and physis. AJR Am J Roentgenol. 1997;169:183-189.

3. Brougham DI, Broughton NS, Cole WG, Menelaus MB. Avascular necrosis following closed reduction of congenital dislocation of the hip. Review of influencing factors and longterm follow-up. J Bone Joint Surg Br. 1990;72:557-562.

4. Carney BT, Clark D, Minter CL. Is the absence of the ossific nucleus prognostic for avascular necrosis after closed reduction of developmental dysplasia of the hip? J Surg Orthop Adv. 2004;1:24-29.

5. Chin MS, Betz BW, Halanski MA. Comparison of hip reduction using magnetic resonance imaging or computed tomography in hip dysplasia. J Pediatr Orthop. 2011;31:525-529.

6. Clarke NM, Jowett AJ, Parker L. The surgical treatment of established congenital dislocation of the hip: results of surgery after planned delayed intervention following the appearance of the capital femoral ossific nucleus. J Pediatr Orthop. 2005;4: 434-439.

7. Cooke SJ, Rees R, Edwards DL, Kiely NT, Evans GA. Ossification of the femoral head at closed reduction for developmental dysplasia of the hip and its influence on the long-term outcome. $J$ Pediatr Orthop B. 2010;1:22-26.

8. Cooper AP, Doddabasappa SN, Mulpuri K. Evidence-based management of developmental dysplasia of the hip. Orthop Clin North Am. 2014;45:341-354.

9. Cooperman DR, Wallensten R, Stulberg SD. Post-reduction avascular necrosis in congenital dislocation of the hip. $J$ Bone Joint Surg Am. 1980;62:247-258.

10. Düppe H, Danielsson LG. Screening of neonatal instability and of developmental dislocation of the hip. A survey of 132,601 living newborn infants between 1956 and 1999. J Bone Joint Surg Br. 2002;84:878-885.

11. Hoellwarth JS, Kim YJ, Millis MB, Kasser JR, Zurakowski D, Matheney TH. Medial versus anterior open reduction for developmental hip dislocation in age-matched patients. J Pediatr Orthop. 2015;1:50-56.

12. Jaramillo D, Villegas-Medina O, Doty DK, Dwek JR, Ransil BJ, Mulkern RV, Shapiro F. Gadolinium-enhanced MR imaging demonstrates abduction-caused hip ischemia and its reversal in piglets. AJR Am J Roentgenol. 1996;166:879-887.

13. Jaramillo D, Villegas-Medina O, Doty DK, Rivas R, Strife K, Dwek JR, Mulkern RV, Shapiro F. Age-related vascular changes in the epiphysis, physis, and metaphysis: normal findings on gadolinium-enhanced MRI of piglets. AJR Am J Roentgenol. 2004; 182:353-360.

14. Jaramillo D, Villegas-Medina O, Laor T, Shapiro F, Millis MB. Gadolinium-enhanced MR imaging of pediatric patients after reduction of dysplastic hips: assessment of femoral head position, factors impeding reduction, and femoral head ischemia. AJR Am J Roentgenol. 1998;170:1633-1637.

15. Kalamchi A, MacEwen GD. Avascular necrosis following treatment of congenital dislocation of the hip. J Bone Joint Surg Am. 1980;62:876-888.

16. Li Y, Xu H, Li J, Yu L, Liu Y, Southern E, Liu H. Early predictors of acetabular growth after closed reduction in late detected developmental dysplasia of the hip. J Pediatr Orthop B. 2015;1:35-39.
17. Luhmann SJ, Bassett GS, Gordon JE, Schootman M, Schoenecker PL. Reduction of a dislocation of the hip due to developmental dysplasia. Implications for the need for future surgery. $J$ Bone Joint Surg Am. 2003;2:239-243.

18. Luhmann SJ, Schoenecker PL, Anderson AM, Bassett GS. The prognostic importance of the ossific nucleus in the treatment of congenital dysplasia of the hip. J Bone Joint Surg Am. 1998;12:1719-1727.

19. Madhu TS, Akula M, Scott BW, Templeton PA. Treatment of developmental dislocation of hip: does changing the hip abduction angle in the hip spica affect the rate of avascular necrosis of the femoral head? J Pediatr Orthop B. 2013;22:184-188.

20. Malvitz TA, Weinstein SL. Closed reduction for congenital dysplasia of the hip. Functional and radiographic results after an average of thirty years. J Bone Joint Surg Am. 1994;76:1777-1792.

21. Murray T, Cooperman DR, Thompson GH, Ballock RT. Closed reduction for treatment of developmental dysplasia of the hip in children. Am J Orthop (Belle Mead NJ). 2007;36:82-84.

22. Narayanan U, Mulpuri K, Sankar WN, Clarke NM, Hosalkar H, Price CT, International Hip Dysplasia Institute Study Group. Reliability of a new radiographic classification for developmental dysplasia of the hip. J Pediatr Orthop. 2014 Sep 26 [Epub ahead of print].

23. Ogden JA. Treatment positions for congenital dysplasia of the hip. J Pediatr. 1975;5:732-734.

24. Roposch A, Odeh O, Doria AS, Wedge JH. The presence of an ossific nucleus does not protect against osteonecrosis after treatment of developmental dysplasia of the hip. Clin Orthop Relat Res. 2011;469:2838-2845.

25. Salter RB, Kostuik J, Dallas S. Avascular necrosis of the femoral head as a complication of treatment for congenital dislocation of the hip in young children: a clinical and experimental investigation. Can J Surg. 1969;12:44-61.

26. Sankar WN, Thomas S, Castañeda P, Hong T, Shore BJ, Kim HK. Study conducted by the International Perthes Study Group. Feasibility and safety of perfusion MRI for Legg-Calvé-Perthes disease. J Pediatr Orthop. 2014;34:679-682.

27. Segal LS, Boal DK, Borthwick L, Clark MW, Localio AR, Schwentker EP. Avascular necrosis after treatment of DDH: the protective influence of the ossific nucleus. J Pediatr Orthop. 1999;19:177-184.

28. Segal LS, Schneider DJ, Berlin JM, Bruno A, Davis BR, Jacobs $\mathrm{CR}$. The contribution of the ossific nucleus to the structural stiffness of the capital femoral epiphysis: a porcine model for DDH. J Pediatr Orthop. 1999;4:433-437.

29. Shipman SA, Helfand M, Moyer VA, Yawn BP. Screening for developmental dysplasia of the hip: a systematic literature review for the US Preventive Services Task Force. Pediatrics. 2006; 117:e557-576.

30. Sibinski M, Synder M, Domzalski M, Grzegorzewski A. Risk factors for avascular necrosis after closed hip reduction in developmental dysplasia of the hip. Ortop Traumatol Rehabil. 2004;1:60-66.

31. Smith BG, Kasser JR, Hey LA, Jaramillo D, Millis MB. Postreduction computed tomography in developmental dislocation of the hip: Part I: Analysis of measurement reliability. J Pediatr Orthop. 1997;17:626-630.

32. Smith BG, Millis MB, Hey LA, Jaramillo D, Kasser JR. Postreduction computed tomography in developmental dislocation of the hip: Part II: Predictive value for outcome. J Pediatr Orthop. 1997; 17:631-636.

33. Tasnavites A, Murray DW, Benson MK. Improvement in acetabular index after reduction of hips with developmental dysplasia. J Bone Joint Surg Br. 1993;5:755-759.

34. Tiderius C, Jaramillo D, Connolly S, Griffey M, Rodriguez DP, Kasser JR, Millis MB, Zurakowski D, Kim YJ. Post-closed reduction perfusion magnetic resonance imaging as a predictor of 
avascular necrosis in developmental hip dysplasia: a preliminary report. J Pediatr Orthop. 2009;29:14-20.

35. Xu M, Gao S, Sun J, Yang Y, Song Y, Han R, Lei G. Predictive values for the severity of avascular necrosis from the initial evaluation in closed reduction of developmental dysplasia of the hip. J Pediatr Orthop B. 2013;3:179-183.

36. Zamzam MM, Kremli MK, Khoshhal KI, Abak AA, Bakarman KA, Alsiddiky AM, Alzain KO. Acetabular cartilaginous angle: a new method for predicting acetabular development in developmental dysplasia of the hip in children between 2 and 18 months of age. J Pediatr Orthop. 2008;5:518-523.

37. Zionts LE, MacEwen GD. Treatment of congenital dislocation of the hip in children between the ages of one and three years. $J$ Bone Joint Surg Am. 1986;6:829-846. 